\title{
Butterfly diversity in relation to nectar food plants from Bhor Tahsil, Pune District, Maharashtra, India
}

\author{
R.K. Nimbalkar ${ }^{1}$, S.K. Chandekar ${ }^{2}$ \& S.P. Khunte ${ }^{3}$ \\ ${ }^{1}$ Department of Zoology, Vinayakrao Patil Mahavidyalaya, Vaijapur, Aurangabad District, Maharashtra 423701, India \\ ${ }^{2}$ Department of Zoology, Annasaheb Magar Mahavidyalaya, Pune, Maharashtra 411028, India \\ ${ }^{3}$ Department of Botany, Mahatma Phule College, Pimpri, Pune, Maharashtra 411017, India \\ Email: rknimbalkar@gmail.com¹, sonalkchandekar@rediffmail.com² (corresponding author)
}

Abstract: Floral attributes are well known to influence nectar feeding butterflies. However, there is paucity of information on food resources of adult butterflies as compared to that of larvae. The present study was carried out from Bhor Tahsil of Pune District, Maharashtra, India, during August 2007 to August 2009. A total of 64 butterfly species were recorded. Family Nymphalidae dominates in the study area, followed by Lycaenidae, Pieridae, Hesperiidae and Papilionidae. Nineteen nectar food plants were identified belonging to 10 plant families. Plants of the Asteraceae family are more used by butterflies as nectar food plants. Visits of butterflies were more frequent to flowers with tubular corollas than to non-tubular ones, to flowers coloured red, yellow, blue and purple than those coloured white and pink and to flower sources available for longer periods in the year. Species abundance reached the peak in the months during August to November. A decline in species abundance was observed from the months December to January and continued up to the end of May. Our findings are important with respect to monitoring butterfly and plant diversity and defining conservation strategies in the Bhor Tahsil.

Keywords: Bhor Tahsil, butterfly diversity, nectar food plants, seasonal distribution.

Date of publication (online): 26 March 2011

Date of publication (print): 26 March 2011

ISSN 0974-7907 (online) | 0974-7893 (print)

Editor: Krushnamegh Kunte

Manuscript details:

Ms \# 02612

Received 23 October 2010

Finally accepted 05 December 2010

Citation: Nimbalkar, R.K., S.K. Chandekar \& S.P. Khunte (2011). Butterfly diversity in relation to nectar food plants from Bhor Tahsil, Pune District, Maharashtra, India. Journal of Threatened Taxa 3(3): 1601-1609.

Copyright: () R.K. Nimbalkar, S.K. Chandekar \& S.P. Khunte 2011 Creative Commons Attribution 3.0 Unported License. JoTT allows unrestricted use of this article in any medium for non-profit purposes, reproduction and distribution by providing adequate credit to the authors and the source of publication.

Acknowledgements: Mrs. S.K. Chandekar expresses a deep sense of gratitude to the Director, Board of College and University Development, Pune University, for Research Grant. We are thankful to Dr. K.A. Subramanian, Mr. A.D. Tiple and Dr. J.S. Wadatkar, for their help in confirming identification of butterfly species. We are also thankful to the Regional Meteorological Centre of Government of India, Mumbai 400099 for providing the rainfall data.

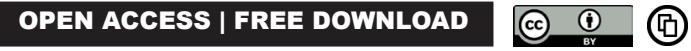

The problems of environmental damage and degradation of natural resources have received increasing attention throughout the country. Pune District is one of the important industrial districts in the state of Maharashtra. The increased industrialization and urbanization has manifold effects on the ecology of this region. It has 14 tahsils, out of which Bhor Tahsil was selected for the study of butterfly diversity in relation to nectar food plants. Bhor Tahsil is famous for historical places, tourist places and dams.

Butterflies are scaled wing insects belonging to the order Lepidoptera of class Insecta. There is an intimate association between butterflies and plants and their lives are exceptionally interlinked (Feltwell 1986), which leads to different patterns in their distribution depending on the availability of their food plants.

Feeding is a significant activity and food may often be the most decisive factor affecting distribution, abundance and movements of animals. In butterflies, this has a special relevance because food and mode of feeding are different in the larval and adult stages (Kunte 2000).

Butterflies and their caterpillars are dependent on specific host plants for foliage, nectar and pollen as their food. Thus butterfly diversity reflects overall plant diversity, especially, that of herbs and shrubs in the given area. Herbs and shrubs start their life cycle in the beginning of the monsoon and complete

This article is part of the peer-reviewed Proceedings of the $3^{\text {rd }}$ Asian Lepidoptera Conservation Symposium (3ALCS-2010) jointly organized by the IUCN SSC South Asian Invertebrate Specialist Group (SAsISG); Department of Zoology, Bharathiar University; Zoo Outreach Organisation and Wildlife Information Liaison Development, held from 25 to 29 October 2010 at Coimbatore, Tamil Nadu, India. http://www.zooreach.org/3alcs2010.html 
it by the end of the postmonsoon season. While some shrubs like Lantana camara shows flowering throught out the year.

Earlier, various workers like Kunte (1997) studied seasonal patterns in butterfly abundance and species diversity in four tropical habitats in the northernWestern Ghats. These four sites are close to Pune City within a radius of $20 \mathrm{~km}$. Kunte (2001) studied the butterfly diversity of Pune City along the human impact gradient; Rane \& Ranade (2004) studied butterflies of Tamhini-Dongarwadi area, Mulshi, Maharashtra; Padhye et al. (2006) studied season and landscape wise distribution of butterflies in Tamhini, northern Western Ghats of India; Sharma (2009) studied the fauna of Bhimashankar Wildlife Sanctuary, Maharashtra; Tiple et al. (2006) studied factors influencing nectar plant resource visits by butterflies and implications for conservation on Amravati University campus. Further, Tiple et al. (2009) investigated butterflyflower morphological interrelationships for 108 butterfly species and 20 plants at Nagpur.

\section{Material and Methods}

Bhor is located $54 \mathrm{~km}$ away from Pune City in a south-westerly direction. It is situated between $18^{\circ} 45^{\prime} \mathrm{N} \& 73^{\circ} 15^{\prime} \mathrm{E}$. It has an elevation of about 591.43m. Bhor Tahsil has an irregular shape, having an area of $892 \mathrm{~km}^{2}$, bordered by Tahsil Khandala of Satara District on the east, Mahad of Raigad District on the west, Wai of Satara District on the south and Velhe, Haveli and Purandar tahsils on the north (Image 1).

The flora of Bhor Tahsil has a great diversity which includes many exotic species. The climate is moist but healthy. The vegetation is mainly of dry deciduous type and scrub type. It is due to moderate and irregular rainfall. The actual rainfall in Bhor Tahsil during the period August 2007 to August 2009 was $2603 \mathrm{~mm}$, as provided by the Regional Meteorological Centre, Mumbai.

The study area was fully explored during August 2007 to August 2009 and then probable areas were decided. To study the seasonal patterns/diversity in butterfly abundance in relation to nectar food plants, the entire year was divided into three seasons. The three seasons of the year are premonsoon from February to May, monsoon from June to September and postmonsoon from October to January. The study area was visited twice in each season during the two years i.e. 2007-2008 and 2008-2009. In the said investigation the selected sites were surveyed mainly between 0730 and $1230 \mathrm{hr}$. Butterfly species were

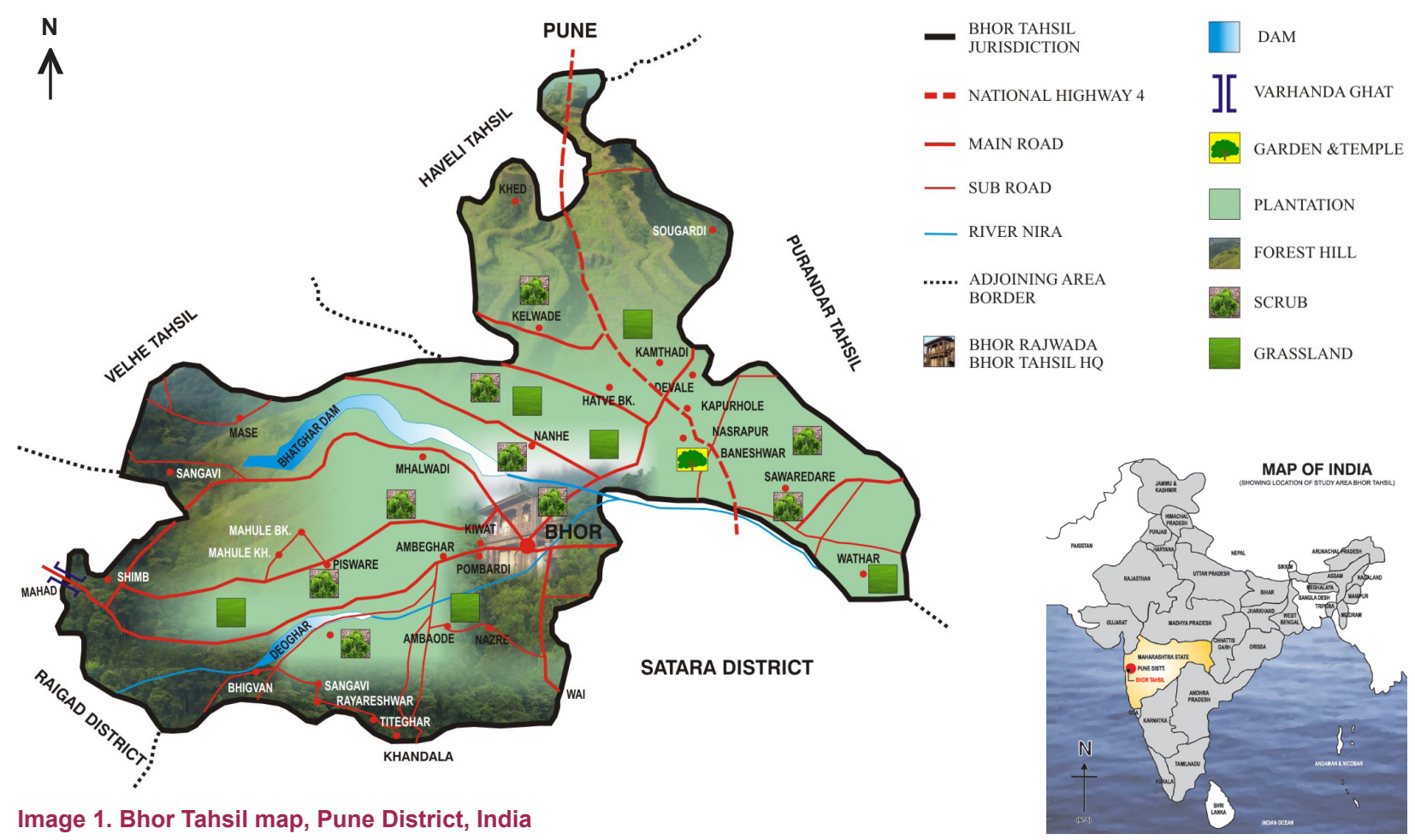


identified directly in the field visually with the help of field guides followed by photography, in difficult cases, rarely by capture. Collection was restricted to those specimens that could not be identified directly. All scientific names follow Varshney (1983) and common English names follow Wynter-Blyth (1957). Classification of butterflies is after Gaonkar (1996). Benthum \& Hooker (1862) system of classification is followed for plants. GPS readings and biotopes of a few sites in Bhor Tahsil area are given in Table 1.

\section{Results}

During the course of study, 64 species of butterflies belonging to five families were recorded in Bhor Tahsil. Out of 64 species, six belong to Papilionidae, eleven to Pieridae, 23 to Nymphalidae, seventeen to Lycaenidae and seven to Hesperiidae. Species belonging to the family Nymphalidae were the most dominant (36\%) followed by Lycaenidae (27\%), Pieridae (17\%), Hesperiidae (11\%) and Papilionidae (9\%).

The status recording was as follows: VC - very common (75-100 sightings), C - common (50-75 sightings), NR - not rare (25-50 sightings), R - rare (5-25 sightings) and VR - very rare (1-5 sightings). Among the 64 species 15 were found very common, 27 species common, 17 species not rare and five species were found rare. None of the species were observed in very rare category from the study area. Six species (Pachliopta hector, Neptis jumbah, Hypolimnas misippus, Lampides boeticus, Euchrysops cnejus and Acytolepis puspa) come under protection of the Indian Wildlife (Protection) Act 1972. Out of the 64 species 27 species were recorded from botanical and nursery gardens, 55 from forest areas, 33 from grasslands, 58 on plantations and 51 from scrub biotope. Results are indicated in Table 2. Nectar food plants of butterfly

Table 1. GPS readings and biotopes of a few sites in Bhor Tahsil area

\begin{tabular}{|c|c|c|c|c|c|}
\hline \multicolumn{2}{|c|}{$\begin{array}{c}\text { Locations in } \\
\text { Bhor Tahsil }\end{array}$} & \multicolumn{3}{|c|}{ GPS Readings } & \multirow{2}{*}{$\begin{array}{c}\text { Type of } \\
\text { Biotope }\end{array}$} \\
\cline { 1 - 5 } & Location & Latitude & Longitude & Altitude & \\
\hline 1 & Baneshwar & $18^{0} 15.406^{\prime}$ & $73^{0} 52.346^{\prime}$ & $655 \mathrm{~m}$ & Garden \\
\hline 2 & Bhor & $18^{0} 45.340^{\prime}$ & $73^{0} 14.601^{\prime}$ & $592 \mathrm{~m}$ & Scrub \\
\hline 3 & Pisavare & $18^{\circ} 07.902^{\prime}$ & $73^{0} 47.68^{\prime}$ & $601 \mathrm{~m}$ & Plantation \\
\hline 4 & Pombardi & $18^{0} 08.467^{\prime}$ & $73^{\circ} 48.926^{\prime}$ & $630 \mathrm{~m}$ & Forest \\
\hline 5 & Wathar & $18^{\circ} 07.781^{\prime}$ & $73^{\circ} 47.636^{\prime}$ & $605 \mathrm{~m}$ & Grassland \\
\hline
\end{tabular}

species and floral characteristics of plants are indicated in Table 3. Mud puddling is usually observed in males. However, females of Hypolimnas bolina and Hypolimnas misippus were also observed while mud puddling (Table 4).

Ten families of plants are used by butterflies as nectar food plants, as recorded from the study area: six plants of the family Asteraceae, two plants of each family Asclepiadaceae, Caesalpiniaceae, Fabaceae and Verbenaceae, while only one plant of each family Amaranthaceae, Apocynaceae, Malvaceae, Rubiaceae and Thymeleaceae. Visits of butterflies were more frequent to flowers of herbs and shrubs rather than to flowers of trees (Table 4).

\section{Discussion and Conclusions}

The species abundance rose from the beginning of the monsoon, from the months June to July and reached a peak in the months from August to November. A decline in species abundance was observed from the months December to January and continued up to the end of May. A previous study (Wynter-Blyth 1956) had identified two seasons as peaks, March-April and October for butterfly abundance in India. However, our finding observed peak period in the months from August to November, in line with the findings of Kunte (2000). Bhusal \& Khanal (2008) reported that there is a significant correlation between species diversity and spring season, indicating the abundances of diverse species was positively affected by approaching warmer days, high relative humidity and more rainfall. These factors help to flourish diverse vegetations, which are vital food sources for many butterfly species. Gutierrez \& Mendez (1995) suggested that the abundance of butterflies is not affected by altitudes but it is more related to the availability of food plants. A similar seasonal variation in species abundance was observed by Prajapati et al. (2000) in Daman of Makawanpur District of central Nepal. Plants have importance in increasing the butterfly diversity and their abundance in the area. In study area, maximum species of butterflies were recorded on plantation biotope, followed by forest and scrub biotope. However, grassland and botanical and nursery gardens are not observed as rich biotopes; heavy grazing pressure on grassland and use of pesticides in gardens have adversely affected diversity of butterflies in these biotopes. The nectar flowering plants visited by 
Table 2. Biotopes, status and seasonal sightings of butterfly species from Bhor Tahsil, Pune District, India

\begin{tabular}{|c|c|c|c|c|c|c|c|c|}
\hline & Common name & Scientific name & Biotopes & Status & \multicolumn{4}{|c|}{ Seasonal sightings } \\
\hline & & & & & Monsoon & $\begin{array}{c}\begin{array}{c}\text { Post- } \\
\text { monsoon }\end{array} \\
\end{array}$ & \begin{tabular}{|c|} 
Pre- \\
monsoon
\end{tabular} & Total \\
\hline \multicolumn{9}{|c|}{ Papilionidae } \\
\hline 1 & Common Bluebottle & Graphium sarpedon Linnaeus & BFP & C & 28 & 12 & 10 & 50 \\
\hline 2 & Tailed Jay & Graphium agamemnon Linnaeus & BGP & C & 35 & 20 & 15 & 70 \\
\hline 3 & Common Mormon & Papilio polytes Linnaeus & BFGP & VC & 42 & 25 & 18 & 85 \\
\hline 4 & Lime Butterfly & Papilio demoleus Linnaeus & BFGPS & C & 30 & 25 & 5 & 60 \\
\hline 5 & Common Rose & Pachliopta aristolochiae Fabricius & BFGPS & C & 28 & 16 & 7 & 51 \\
\hline 6 & Crimson Rose ${ }^{*}$ & Pachliopta hector Linnaeus & BFPS & c & 29 & 15 & 6 & 50 \\
\hline \multicolumn{9}{|c|}{ Pieridae } \\
\hline 7 & Three Spot Grass Yellow & Eurema blanda Boisduval & FS & NR & 20 & 15 & 5 & 40 \\
\hline 8 & Small Grass Yellow & Eurema brigitta Cramer & BFGPS & VC & 50 & 28 & 18 & 96 \\
\hline 9 & Common Grass Yellow & Eurema hecabe Linnaeus & BFGPS & VC & 48 & 22 & 27 & 97 \\
\hline 10 & Spotless Grass Yellow & Eurema laeta Boisduval & FGPS & C & 30 & 15 & 10 & 55 \\
\hline 11 & Common Emigrant & Catopsilia pomona Fabricius & BFGPS & VC & 45 & 35 & 19 & 99 \\
\hline 12 & Mottled Emigrant & Catopsilia pyranthe Linnaeus & BFGPS & VC & 42 & 24 & 12 & 78 \\
\hline 13 & White Orange Tip & Ixias marianne Cramer & BFPS & C & 17 & 30 & 15 & 62 \\
\hline 14 & Common Gull & Cepora nerissa Fabricius & FGPS & C & 35 & 25 & 15 & 75 \\
\hline 15 & Common Jezebel & Delias eucharis Drury & FPS & C & 25 & 20 & 8 & 53 \\
\hline 16 & Psyche & Leptosia nina Fabricius & FGPS & $\mathrm{R}$ & 15 & 12 & 0 & 27 \\
\hline 17 & Pioneer & Belenois aurota Fabricicus & BFGPS & VC & 39 & 37 & 19 & 95 \\
\hline \multicolumn{9}{|c|}{ Nymphalidae } \\
\hline 18 & Blue Tiger & Tirumala limniace Cramer & FPS & C & 35 & 25 & 6 & 66 \\
\hline 19 & Striped Tiger & Danaus genutia Cramer & FGPS & C & 25 & 35 & 8 & 68 \\
\hline 20 & Plain Tiger & Danaus chrysippusLinnaeus & BFGPS & VC & 40 & 35 & 25 & 100 \\
\hline 21 & Glassy Tiger & Parantica aglea Stoll & FGPS & C & 26 & 20 & 5 & 51 \\
\hline 22 & Common Indian Crow & Euploea core Cramer & BFGPS & VC & 37 & 24 & 20 & 81 \\
\hline 23 & Common Nawab & Polyura athamas Drury & FPS & $\mathrm{R}$ & 10 & 6 & 2 & 18 \\
\hline 24 & Black Rajah & Charaxes Solon Fabricius & FPS & $\mathrm{R}$ & 12 & 7 & 3 & 22 \\
\hline 25 & Common Evening Brown & Melanitis leda Linnaeus & FPS & VC & 43 & 32 & 11 & 86 \\
\hline 26 & Common Three Ring & Ypthima asterope Klug & FGS & NR & 17 & 9 & 4 & 30 \\
\hline 27 & Common Five Ring & Ypthima baldus Fabricius & FS & NR & 21 & 9 & 5 & 35 \\
\hline 28 & Tawny Coster & Acraea violae Fabricius & BGPS & C & 33 & 25 & 14 & 72 \\
\hline 29 & Common Leopard & Phalanta phalantha Drury & FGPS & VC & 38 & 26 & 26 & 90 \\
\hline 30 & $\begin{array}{l}\text { Chestnut Streaked } \\
\text { Sailer* }\end{array}$ & Neptis jumbah Moore & FPS & NR & 18 & 9 & 7 & 34 \\
\hline 31 & Angled Castor & Ariadne ariadne Linnaeus & BGPS & VC & 40 & 31 & 13 & 84 \\
\hline 32 & Common Castor & Ariadne merione Cramer & BGPS & C & 29 & 17 & 12 & 58 \\
\hline 33 & Painted Lady & Vanessa cardui Linnaeus & GPS & C & 29 & 25 & 7 & 61 \\
\hline 34 & Blue Pansy & Junonia orithiya Linnaeus & FGPS & C & 27 & 21 & 17 & 65 \\
\hline 35 & Yellow Pansy & Junonia hierta Fabricius & FGPS & C & 24 & 19 & 10 & 53 \\
\hline 36 & Chocolate Pansy & Junonia iphita Cramer & BFGPS & C & 32 & 16 & 8 & 56 \\
\hline 37 & Grey Pansy & Junonia atlites Linnaeus & BFPS & C & 18 & 24 & 8 & 50 \\
\hline 38 & Lemon Pansy & Junonia lemonias Linnaeus & BFGPS & VC & 48 & 30 & 22 & 100 \\
\hline 39 & Great Eggfly & Hypolimnas bolina Linnaeus & BFPS & VC & 36 & 28 & 18 & 82 \\
\hline 40 & Danaid Eggfly* & Hypolimnas misippus Linnaeus & BFPS & VC & 44 & 25 & 7 & 76 \\
\hline
\end{tabular}




\begin{tabular}{|c|c|c|c|c|c|c|c|c|}
\hline & Common name & Scientific name & Biotopes & Status & \multicolumn{4}{|c|}{ Seasonal sightings } \\
\hline & & & & & Monsoon & $\begin{array}{c}\text { Post- } \\
\text { monsoon }\end{array}$ & $\begin{array}{c}\text { Pre- } \\
\text { monsoon }\end{array}$ & Total \\
\hline \multicolumn{9}{|c|}{ Lycaenidae } \\
\hline 41 & Silver Streak Blue & Iraota timoleon Stoll & FP & NR & 16 & 7 & 5 & 28 \\
\hline 42 & Large Guava Blue & Deudorix perse Hewitson & FP & NR & 11 & 8 & 6 & 25 \\
\hline 43 & Angled Pierrot & Caleta caleta Hewitson & $\mathrm{F}$ & NR & 20 & 10 & 0 & 30 \\
\hline 44 & Banded Blue Pierrot & Discolampa ethion Westwood & FPS & NR & 16 & 12 & 0 & 28 \\
\hline 45 & Zebra Blue & Leptotes plinius Fabricius & FGPS & $\mathrm{C}$ & 31 & 19 & 4 & 54 \\
\hline 46 & Common Cerulean & Jamides celenoCramer & FP & VC & 34 & 25 & 20 & 79 \\
\hline 47 & Forget-me-not & Catochrysops strabo Fabricius & FPS & NR & 13 & 9 & 6 & 28 \\
\hline 48 & Pea Blue* & Lampides boeticus Linnaeus & BFGS & $\mathrm{C}$ & 20 & 30 & 18 & 68 \\
\hline 49 & Dark Grass Blue & Zizeeria Karsandra Moore & FGPS & NR & 20 & 14 & 7 & 41 \\
\hline 50 & Pale Grass Blue & Pseudozizeeria maha Kollar & BGPS & NR & 17 & 8 & 5 & 30 \\
\hline 51 & Tiny Grass Blue & Zizula hylax Fabricius & BFGPS & $\mathrm{C}$ & 37 & 20 & 15 & 72 \\
\hline 52 & Red Pierrot & Talicada nyseus Guerin -Meneville & BPS & NR & 21 & 14 & 10 & 45 \\
\hline 53 & Gram Blue* & Euchrysops cnejus Fabricius & FGPS & NR & 18 & 12 & 7 & 37 \\
\hline 54 & Common Hedge Blue* & Acytolepis puspa Horsfield & FPS & $\mathrm{C}$ & 32 & 19 & 5 & 56 \\
\hline 55 & Plains Cupid & Chilades pandava Horsfield & GPS & NR & 19 & 9 & 3 & 31 \\
\hline 56 & Lime Blue & Chilades laius Stoll & $\mathrm{BP}$ & $\mathrm{C}$ & 36 & 25 & 10 & 71 \\
\hline 57 & Plum Judy & Abisara echerius Stoll & FPS & $\mathrm{C}$ & 19 & 29 & 10 & 58 \\
\hline \multicolumn{9}{|c|}{ Hesperiidae } \\
\hline 58 & Fulvous Pied Flat & Pseudocoladenia dan Fabricius & $\mathrm{FP}$ & $\mathrm{C}$ & 29 & 17 & 7 & 53 \\
\hline 59 & Dark Palm Dart & Telicota ancilla Herrich-Schaffer & FS & NR & 17 & 7 & 2 & 26 \\
\hline 60 & Rice Swift & Borbo cinnara Wallace & FPS & $\mathrm{C}$ & 31 & 19 & 4 & 54 \\
\hline 61 & Conjoined Swift & Pelopidas conjuncta Herrich-Schaffer & $\mathrm{FP}$ & $\mathrm{R}$ & 8 & 5 & 1 & 14 \\
\hline 62 & Vindhyan Bob & Arnetta vindhiana Moore & FP & $\mathrm{R}$ & 17 & 8 & 0 & 25 \\
\hline 63 & Chestnut Bob & lambrix salsala Moore & $\mathrm{FP}$ & NR & 22 & 23 & 5 & 50 \\
\hline 64 & Grass Demon & Udaspes folus Cramer & $\mathrm{FP}$ & NR & 20 & 10 & 5 & 35 \\
\hline
\end{tabular}

Biotopes: B - Botanical and Nursery Garden; F - Forest; G - Grassland; P - Plantation; S - Scrub

Status: C - Common; VC - Very Common; R - Rare; NR - Not Rare; VR - Very Rare; * - Scheduled species

butterflies, as observed in our findings, namely Carissa congesta, Asclepias curassavica, Calotropis gigantea, Senecio bombayensis, Vernonia divergens, Wedelia uticaefolia, Zinnia eleganas, Cassia auriculata, Urena lobata, Mussaenda glabrata and Gnidia glauca are not reported by Tiple et al. $(2006,2009)$ in their study area of Amravati University Campus and Nagpur, Central India, respectively. The herbs from the study area namely Celosia argentea, Tridax procumbens and Tephrosia purpurea are more used by butterflies, probably due to the fact that the flowering period of these herbs is throughout the year. The shrubs namely Calotropis gigantea and Lantana camara also have a flowering period throughout the year, so they are more used by butterflies as their food plants. A few species of butterflies were observed feeding on either animal droppings or on ripe fruits or while mud puddling (Table 4). Mud puddling is usually observed in males, but in our findings females of Hypolimnas bolina and Hypolimnas misippus butterfly species were observed doing mud puddling. Mathew \& Binoy (2002) reported that females of Appias albina darada were found to be very much active in mud puddling. The requirement of more water and salt could be the reason for this.

Monitoring and mapping biodiversity is the first step in systematic conservation planning (Margules \& Pressey 2000). In the study area, events like grazing pressure, influx of tourists, construction of highways, use of pesticides and change in land use pattern, are mainly responsible for diversity loss of both butterflies and plants. Members from family Lycaenidae largely feed on grasses and cattle grazing affected their diversity 
Table 3. Nectar food plants of buttrerfly species and floral characteristics of plants from Bhor Tahsil, Pune District, India

\begin{tabular}{|c|c|c|c|c|c|}
\hline Family / Botanical name & Habit & $\begin{array}{l}\text { Flowering } \\
\text { period }\end{array}$ & Flower colour & Corolla shape & $\begin{array}{l}\text { Flower } \\
\text { abundance }\end{array}$ \\
\hline \multicolumn{6}{|l|}{ Amaranthaceae } \\
\hline Celosia argentea L. & Herb & Aug-Feb & Pink, White & NT & $\mathrm{D}$ \\
\hline \multicolumn{6}{|l|}{ Apocynaceae } \\
\hline Carissa congesta Wight & Shrub & Apr-Jun & White & $\mathrm{T}$ & M \\
\hline \multicolumn{6}{|l|}{ Asclepiadaceae } \\
\hline Asclepias curassavica L. & Undershrub & Jan-Dec & Red, Yellow & NT & $\mathrm{D}$ \\
\hline Calotropis gigantea (L.) Ait. & Shrub & Oct-July & Purple, White & NT & M \\
\hline \multicolumn{6}{|l|}{ Asteraceae } \\
\hline Cosmos bipinnatus Cav. & Herb & Aug-Nov & Orange, Yellow & $\mathrm{T}$ & $\mathrm{D}$ \\
\hline Senecio bombayensis Balakr. & Herb & Aug-Dec & Yellow & $\mathrm{T}$ & $\mathrm{D}$ \\
\hline Tridax procumbens $\mathrm{L}$. & Herb & Jan-Dec & Yellowish White & $\mathrm{T}$ & $\mathrm{D}$ \\
\hline Vernonia divergens (Roxb.) Edgew. & Shrub & Nov-Apr & Purple, White & $\mathrm{T}$ & $\mathrm{D}$ \\
\hline Wedelia urticaefolia DC & Herb & Aug-Sep & Yellow & $\mathrm{T}$ & $\mathrm{D}$ \\
\hline Zinnia eleganas Jacq. & Herb & Aug-Dec & Pink, Yellow & $\mathrm{T}$ & $\mathrm{D}$ \\
\hline \multicolumn{6}{|l|}{ Caesalpiniaceae } \\
\hline Bauhinia purpurea L. & Tree & Sep-Jan & Purple & NT & $\mathrm{S}$ \\
\hline Cassia auriculata L. & Shrub & Jan-Jul & Yellow & NT & M \\
\hline \multicolumn{6}{|l|}{ Fabaceae } \\
\hline Tephrosia purpurea (L.) Pers. & Undershrub & Jan-Dec & Rosy Purple & NT & M \\
\hline Crotalaria juncea L. & Herb & Oct-Jan & Yellow & NT & $M$ \\
\hline \multicolumn{6}{|l|}{ Malvaceae } \\
\hline Urena lobata L. & Shrub & Jul-Dec & Pink & $\mathrm{T}$ & $S$ \\
\hline \multicolumn{6}{|l|}{ Rubiaceae } \\
\hline $\begin{array}{l}\text { Mussaenda glabrata (Hook. F.) Hutch. ex } \\
\text { Gamble }\end{array}$ & Shrub & Feb-Sep & Orange-Red & $\mathrm{T}$ & $S$ \\
\hline \multicolumn{6}{|l|}{ Thymeleaceae } \\
\hline Gnidia glauca (Fresen.) Gilg. & Shrub & Oct-Jun & Bright Yellow & $\mathrm{T}$ & $\mathrm{D}$ \\
\hline \multicolumn{6}{|l|}{ Verbenaceae } \\
\hline Lantana camara L. & Shrub & Jan-Dec & Orange-Red & $\mathrm{T}$ & $\mathrm{D}$ \\
\hline Vitex negundo L. & Shrub & Jan-Jul & Bluish-Purple & $\mathrm{T}$ & $M$ \\
\hline
\end{tabular}

and abundance. In the United Kingdom grazing by cattle and sheep has been practiced as a management tool (Pollard 1991) and there is ample scope for such practices in India. A total of five species of butterflies from the study area are designated rare while describing their status and justifies its inclusion in scheduled list suggesting the need for strict conservation measures (Table 2). As reported by Kunte (2000), an objective revision of the scheduled list will be very useful in providing appropriate and adequate legal protection to Indian butterflies.

Our findings are more important for monitoring butterfly diversity and nectar food plant diversity to improve the ecological utility of butterflies as indicator taxa and pollinating agents and defining conservation strategies in the study area.

\section{REFERENCES}

Benthum, G. \& J.D. Hooker (1862-1883). Genera Plantarum Vol. I, II, III. London, 1040, 1279, 1258pp.

Bhusal, D.R. \& B. Khanal (2008). Seasonal and Altitudinal Diversity of Butterflies in Eastern Siwalik of Nepal. Journal of the Natural History Museum 23: 82-87.

Feltwell, J. (1986). The Natural History of Butterflies. Groom Helem Ltd., Provident House, Bureel Row, Beckenham 
Table 4. Nectar food plants and other food sources of butterfly species observed from study area Bhor Tahsil, Pune District, india

\begin{tabular}{|c|c|c|c|}
\hline & Common name & Scientific name & Scientific name of nectar food plant / other source \\
\hline \multicolumn{4}{|c|}{ Papilionidae } \\
\hline 1 & Common Bluebottle & Graphium sarpedon Linnaeus & Cosmos bipinnatus, Zinnia eleganas \\
\hline 2 & Tailed Jay & Graphium agamemnon Linnaeus & Lantana camara \\
\hline 3 & Common Mormon & Papilio polytes Linnaeus & Cosmos bipinnatus, Lantana camara \\
\hline 4 & Lime Butterfly & Papilio demoleus Linnaeus & $\begin{array}{l}\text { Lantana camara, Mussaenda galbrata, Tephrosia purpurea, Tridax } \\
\text { procumbens }\end{array}$ \\
\hline 5 & Common Rose & Pachliopta aristolochiae Fabricius & Lantana camara \\
\hline 6 & Crimson Rose & Pachliopta hector Linnaeus & Lantana camara, Tridax procumbens \\
\hline \multicolumn{4}{|c|}{ Pieridae } \\
\hline 7 & Three Spot Grass Yellow & Eurema blanda Boisduval & Tephrosia purpurea, Tridax procumbens \\
\hline 8 & Small Grass Yellow & Eurema brigitta Cramer & Lantana camara, Urena lobata, Zinnia elegans \\
\hline 9 & Common Grass Yellow & Eurema hecabe Linnaeus & $\begin{array}{l}\text { Celosia argenta, Lantana camara, Tephrosia purpurea, Tridax } \\
\text { procumbens }\end{array}$ \\
\hline 10 & Spotless Grass Yellow & Eurema laeta Boisduval & Celosia argenta, Lantana camara, Tridax procumbens \\
\hline 11 & Common Emigrant & Catopsilia pomona Fabricius & $\begin{array}{l}\text { Cassia auriculata, Lantana camara, Tephrosia purpurea, Tridax } \\
\text { procumbens, Wedelia uticaefolia }\end{array}$ \\
\hline 12 & Mottled Emigrant & Catopsilia pyranthe Linnaeus & Lantana camara, Tridax procumbens \\
\hline 13 & White Orange Tip & Ixias marianne Cramer & Calotropis gigantea, Tridax procumbens \\
\hline 14 & Common Gull & Cepora nerissa Fabricius & Asclepias curassavica, Lantana camara, Tridax procumbens \\
\hline 15 & Common Jezebel & Delias eucharis Drury & Celosia argenta, Lantana camara \\
\hline 16 & Psyche & Leptosia nina Fabricius & Tridax procumbens \\
\hline 17 & Pioneer & Belenois aurota Fabricicus & Calotropis gigantea, Lantana camara, Tridax procumbens \\
\hline \multicolumn{4}{|c|}{ Nymphalidae } \\
\hline 18 & Blue Tiger & Tirumala limniace Cramer & Crotalaria juncea, Lantana camara, Tridax procumbens \\
\hline 19 & Striped Tiger & Danaus genutia Cramer & $\begin{array}{l}\text { Celosia argentea, Crotalaria juncea, Lantana camara, Tridax } \\
\text { procumbens, Sencio bombayenesis }\end{array}$ \\
\hline 20 & Plain Tiger & Danaus chrysippusLinnaeus & $\begin{array}{l}\text { Crotalaria juncea, Lantana camara, Tridax procumbens, Vitex } \\
\text { negundo, Zinnia elegans }\end{array}$ \\
\hline 21 & Glassy Tiger & Parantica aglea Stoll & Crotalaria juncea, Lantana camara, Zinnia elegans \\
\hline 22 & Common Indian Crow & Euploea core Cramer & $\begin{array}{l}\text { Celosia argentea, Cosmos sulphureus, Lantana camara, Tridax } \\
\text { procumbens, Zinnia elegans }\end{array}$ \\
\hline 23 & Common Nawab & Polyura athamas Drury & On animal droppings \\
\hline 24 & Black Rajah & Charaxes Solon Fabricius & On animal droppings, on over-ripe fruits \\
\hline 25 & Common Evening Brown & Melanitis leda Linnaeus & Tridax procumbens \\
\hline 26 & Common Three Ring & Ypthima asterope Klug & Celosia argentea, Tridax procumbens \\
\hline 27 & Common Five Ring & Ypthima baldus Fabricius & Celosia argentea, Tridax procumbens \\
\hline 28 & Tawny Coster & Acraea violae Fabricius & Lantana camara, Tridax procumbens, Vitex negundo \\
\hline 29 & Common Leopard & Phalanta phalantha Drury & Celosia argentea, Lantana camara, Tridax procumbens \\
\hline 30 & Chestnut Streaked Sailer & Neptis jumbah Moore & Tridax procumbens \\
\hline 31 & Angled Castor & Ariadne ariadne Linnaeus & Lantana camara, Tridax procumbens \\
\hline 32 & Common Castor & Ariadne merione Cramer & Lantana camara, Tridax procumbens \\
\hline 33 & Painted Lady & Vanessa cardui Linnaeus & Carissa congesta, Gnidia glauca, Lantana camara, Tridax procumbens \\
\hline 34 & Blue Pansy & Junonia orithiya Linnaeus & Celosia argentea, Lantana camara, Tridax procumbens \\
\hline 35 & Yellow Pansy & Junonia hierta Fabricius & Celosia argentea, Lantana camara, Tephrosia purpurea \\
\hline 36 & Chocolate Pansy & Junonia iphita Cramer & Tephrosia purpurea \\
\hline 37 & Grey Pansy & Junonia atlites Linnaeus & Celosia argentea, Cosmos sulphureus, Tridax procumbens \\
\hline 38 & Lemon Pansy & Junonia lemonias Linnaeus & Celosia argentea, Tephrosia purpurea, Tridax procumbens \\
\hline
\end{tabular}




\begin{tabular}{|c|c|c|c|}
\hline & Common name & Scientific name & Scientific name of nectar food plant / other source \\
\hline 39 & Great Eggfly & Hypolimnas bolina Linnaeus & Bauhinia purpurea, Celosia argentea, Lantana camara \\
\hline 40 & Danaid Eggfly & Hypolimnas misippus Linnaeus & $\begin{array}{l}\text { Asclepias curassavica, Celosia argentea, Lantana camara, Zinnia } \\
\text { elegans }\end{array}$ \\
\hline \multicolumn{4}{|c|}{ Lycaenidae } \\
\hline 41 & Silver Streak Blue & Iraota timoleon Stoll & Observed doing Mud Puddling \\
\hline 42 & Large Guava Blue & Deudorix perse Hewitson & Observed doing Mud Puddling \\
\hline 43 & Angled Pierrot & Caleta caleta Hewitson & Observed doing Mud Puddling \\
\hline 44 & Banded Blue Pierrot & Discolampa ethion Westwood & Lantana camara \\
\hline 45 & Zebra Blue & Leptotes plinius Fabricius & $\begin{array}{l}\text { Celosia argentea, Lantana camara, Tephrosia purpurea, Tridax } \\
\text { procumbens }\end{array}$ \\
\hline 46 & Common Cerulean & Jamides celenoCramer & Celosia argentea, Tephrosia purpurea, Tridax procumbens \\
\hline 47 & Forget-me-not & Catochrysops strabo Fabricius & Celosia argentea \\
\hline 48 & Pea Blue & Lampides boeticus Linnaeus & Celosia argentea \\
\hline 49 & Dark Grass Blue & Zizeeria Karsandra Moore & Lantana camara \\
\hline 50 & Pale Grass Blue & Pseudozizeeria maha Kollar & Tephrosia purpurea \\
\hline 51 & Tiny Grass Blue & Zizula hylax Fabricius & Lantana camara \\
\hline 52 & Red Pierrot & Talicada nyseus Guerin-Meneville & Tridax procumbens \\
\hline 53 & Gram Blue & Euchrysops cnejus Fabricius & Lantana camara \\
\hline 54 & Common Hedge Blue & Acytolepis puspa Horsfield & Tephrosia purpurea \\
\hline 55 & Plains Cupid & Chilades pandava Horsfield & Tridax procumbens \\
\hline 56 & Lime Blue & Chilades laius Stoll & Urena lobata \\
\hline 57 & Plum Judy & Abisara echerius Stoll & On animal droppings \\
\hline \multicolumn{4}{|c|}{ Hesperiidae } \\
\hline 58 & Fulvous Pied Flat & Pseudocoladenia dan Fabricius & Lantana camara \\
\hline 59 & Dark Palm Dart & Telicota ancilla Herrich-Schaffer & Lantana camara, Tridax procumbens \\
\hline 60 & Rice Swift & Borbo cinnara Wallace & Celosia argentea, Tephrosia purpurea, Tridax procumbens \\
\hline 61 & Conjoined Swift & $\begin{array}{l}\text { Pelopidas conjuncta Herrich- } \\
\text { Schaffer }\end{array}$ & Celosia argentea, Tridax procumbens \\
\hline 62 & Vindhyan Bob & Arnetta vindhiana Moore & Lantana camara \\
\hline 63 & Chestnut Bob & lambrix salsala Moore & Zinnia elegans \\
\hline 64 & Grass Demon & Udaspes folus Cramer & Lantana camera \\
\hline
\end{tabular}

Kent BR3 1AT, 133pp.

Gaonkar, H. (1996). Butterflies of the Western Ghats, India (including Sri Lanka). A biodiversity assessment of a threatened mountain system. Report to the Centre for Ecological Sciences, Bangalore.

Gutierrez, D. \& R. Mendez (1995). Phenology of butterflies in a mountain area in northen Iberian Peninsual. Ecography 18: 209-2196.

Kunte, K. (1997). Seasonal patterns in butterfly abundance and species diversity in four tropical habitats in northern Western Ghats. Journal of Bioscience 22(5): 593-603.

Kunte, K. (2000). Butterflies of Peninsular India.University Press, Hydrabad, India, 254pp.

Kunte, K. (2001). Butterfly diversity of Pune City along the human impact gradient. Journal of Ecological Society 1314: $40-45$.

Margules, C.R. \& R.L. Pressey (2000). Systematic conservation planning. Nature 405: 243-253.

Mathew, G. \& C.F. Binoy (2002). Migration of butterflies (Lepidoptera: Rhopalocera) in the New Amarambalam Reserve Forest of the Nilgiri Biosphere Reserve. Zoos' Print Journal 17(8): 844-847.

Padhye, A.D., N. Dahanukar, M. Paingankar, M. Deshpande \& D. Deshpande (2006). Season and landscape wise distribution of butterflies in Tamhini, north-western Ghats, India. Zoos' Print Journal 21(3): 2175-2181.

Pollard, E. (1991). Monitoring Butterfly numbers, pp. 87-111. In: Goldsmith, B. (ed.) Monitoring for Conservation and Ecology. Chapman and Hall.

Prajapati, B., U. Shrestha \& A.S. Tamrakar (2000). Diversity of butterfly in Daman area of Makawanpur District, Central Nepal. Nepal Journal of Science \& Technology 2: 71-76.

Rane, N.S. \& S.P. Ranade (2004). Butterflies of TamhiniDongarwadi area, Mulshi, Maharashtra. Zoos' Print Journal 
19(3): 1411-1413.

Sharma, R.M. (2009). Insecta: Lepidoptera: Rhopalocera and Grypocera. Fauna of Bhimashankar Wildlife Sanctuary, Conservation Area Series 42: 257-262.

Tiple, A.D. \& A.M. Khurad (2009). Butterflies recorded from Nagpur, central India. Bionotes 11(4): 130-131.

Tiple, A.D., A.M. Khurad \& R.L.H. Dennis (2009). Adult butterfly feeding - nectar flower associations: constraints of taxonomic affiliation, butterfly and nectar flower morphology. Journal of Natural History 13/14: 855-884.

Varshney, R.K. (1983). Index Rhopalocera Indica Part II. Common names of butterflies from India and neighbouring countries. Records of Zoological Survey of India, Occassional Paper No. 47: 1-49.

Wynter-Blyth, M.A. (1957). Butterflies of the Indian Region. Bombay Natural History Society, Mumbai, 523pp. 